\title{
Multiple allosteric effectors control the affinity of DasR for its target sites
}

\author{
Elodie Tenconi ${ }^{\text {a }}$, Mia Urem ${ }^{\text {b }}$, Magdalena A. Świątek-Połatyńska ${ }^{\text {b }}$, Fritz Titgemeyer ${ }^{\text {c }}$, \\ Yves A. Muller ${ }^{\mathrm{d}}$, Gilles P. van Wezel ${ }^{\mathrm{b}}$, Sébastien Rigali ${ }^{\mathrm{a}}$, \\ ${ }^{a}$ Center for Protein Engineering, Institut de chimie B6a, University of Liège, B-4000 Liège, Belgium \\ ${ }^{\mathrm{b}}$ Molecular Biotechnology, Institute of Biology Leiden, Leiden University, Sylviusweg 72, P.O. Box 9502, 2300 RA Leiden, The Netherlands \\ ${ }^{c}$ Department of Oecotrophologie, Münster University of Applied Sciences, Corrensstr. 25, 48149 Münster, Germany \\ ${ }^{\mathrm{d}}$ Lehrstuhl für Biotechnik, Department of Biology, Friedrich-Alexander University Erlangen-Nuremberg, Henkestrasse 91, D-91052 Erlangen, Germany
}

\section{A R T I C L E I N F O}

\section{Article history:}

Received 9 June 2015

Accepted 24 June 2015

Available online 26 June 2015

\section{Keywords:}

Allosteric effector

Ligand

DNA-protein interaction

Gene expression regulation

Nacetylglucosamine

\begin{abstract}
A B S T R A C T
The global transcriptional regulator DasR connects N-acetylglucosamine (GlcNAc) utilization to the onset of morphological and chemical differentiation in the model actinomycete Streptomyces coelicolor. Previous work revealed that glucosamine-6-phosphate (GlcN-6P) acts as an allosteric effector which disables binding by DasR to its operator sites (called dre, for DasR responsive element) and allows derepression of DasR-controlled/GlcNAc-dependent genes. To unveil the mechanism by which DasR controls S. coelicolor development, we performed a series of electromobility shift assays with histidinetagged DasR protein, which suggested that N-acetylglucosamine-6-phosphate (GlcNAc-6P) could also inhibit the formation of DasR-dre complexes and perhaps even more efficiently than GlcN-6P. The possibility that GlcNAc-6P is indeed an efficient allosteric effector of DasR was further confirmed by the high and constitutive activity of the DasR-repressed nagKA promoter in the nagA mutant, which lacks GlcNAc-6P deaminase activity and therefore accumulates GlcNAc-6P. In addition, we also observed that high concentrations of organic or inorganic phosphate enhanced binding of DasR to its recognition site, suggesting that the metabolic status of the cell could determine the selectivity of DasR in vivo, and hence its effect on the expression of its regulon.
\end{abstract}

๑) 2015 Elsevier Inc. All rights reserved.

\section{Introduction}

The transcriptional repressor DasR is a member of the GntR/ HutC family of transcriptional regulators [1] whose core regulon in actinomycetes includes genes involved in the catabolism of $\mathrm{N}$ acetylglucosamine (GlcNAc) and its polymer chitin [2-6]. 'Das' stands for Defect in aerial hyphae and spore formation which refers to the impaired sporulation phenotype exhibited by the dasR null mutants of Streptomyces griseus [7] and Streptomyces coelicolor [2]. The observed developmental defect of dasR mutants fits with a regulon that extends well beyond GlcNAc and chitin utilization genes $[8,9]$, such as gene clusters involved in the production of antibiotics prodiginines and actinorhodin [10] and the desferrioxamine siderophores [11] which are essential for proper sporulation of $S$. coelicolor $[12,13]$. The identification of the DasR regulon and

\footnotetext{
* Corresponding author.

E-mail address: srigali@ulg.ac.be (S. Rigali).
}

the functional characterization of the genes it controls allows the description of the very first complete signalling cascade from extracellular signal transport to the control of secondary metabolite production in the industrially important Streptomyces genus [10].

In the current model of the DasR-controlled and GlcNAcinduced pathways, glucosamine-6-phosphate (GlcN-6P) is the key intracellular molecule sensed by DasR and able to modulate its DNA-binding ability in response to transport and phosphorylation of extracellular GlcNAc by the phosphotransferase system

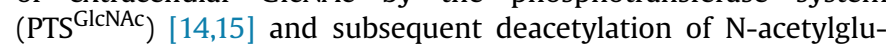
cosamine-6-phosphate (GlcNAc-6P) into GlcN-6P by the GlcNAc-6P deacetylase NagA. Indeed, electromobility gel shift assays (EMSAs) with pure histidine-tagged DasR and DNA probes containing DasR responsive elements (dre) revealed that GlcN-6P was able to inhibit DNA binding by DasR to its recognition site [2]. EMSAs performed with DasR of Saccharopolyspora erythraea $\left(\right.$ DasR $^{\text {sery }}$ ) also revealed that GlcN-6P was able to modulate its DNA-binding capability though in a different way than the simple dissociation of the 
regulatory protein from its target dre observed for DasR of $S$. coelicolor (DasR ${ }^{s c o}$ ) as, instead, multiple retarded bands were observed upon incubation of DasR ${ }^{\text {sery }}$ with GlcN-6P [6]. In Bacilli, the GntR/HutC family NagR (formerly named YvoA [16]) is the DasR orthologue which appears to only control genes for GlcNAc transport and phosphorylation by the PTS (nagP), and the genes for its subsequent deacetylation into GlcN-6P (nagA), and GlcN-6P deamination and isomerization into fructose-6-phosphate (nagB) [17]. The first attempt to identify the allosteric effector of NagR revealed a similar inhibitory effect of GlcN-6P on the DNA-binding ability of NagR as described for DasR ${ }^{S C O}$ [17]. Later on, footprinting assays and EMSAs performed by Gaugé and colleagues did not show any effect of GlcN-6P and a very minor effect of GlcNAc-6P on the DNA-binding ability of NagR [18]. In contrast, the crystal structures of NagR in complex with GlcN-6-P and GlcNAc-6-P suggests that both molecules similarly displace the NagR-DNAbinding domains and therefore should similarly affect the DNAbinding ability of the regulator [19].

Recently, we have shown via genome-wide systems biology analysis that DasR can also bind to genes that do not contain a dre site in their promoter region, in particular during a later stage of its life cycle $[8,9]$. How DasR recognizes such alternative sites, designated Class II (as opposed to Class I for genes controlled via a canonical dre), is yet unclear but presumably requires a co-repressor and/or an effector molecule.

Overall, the compilation of the in vitro data and crystallographic analyses performed with DasR/NagR orthologues of S. coelicolor, Sac. erythraea and Bacillus subtilis, still does not provide a definitive and clear picture on either the nature of the effector (GlcNAc-6P and/or GlcN-6P) or on its allosteric effect (loss of DNA-binding, increased binding, DNA-bending, and/or no effect). In this work we present in vitro and in vivo studies that suggest that DNA binding by DasR is affected by multiple ligands, whereby the metabolic status of the cell modulates the recognition by DasR of its target sites.

\section{Material and methods}

\subsection{Bacterial strains and culture conditions}

All S. coelicolor and Escherichia coli strains used in this study are listed in supplementary Table S1. All routine Streptomyces and E. coli techniques and media are described in the Streptomyces manual [20] and in Sambrook et al. (2001) [21].

\subsection{Inactivation of SCO4284 (nagA) by Tn5062 insertion}

Derivatives of cosmid D95A carrying Tn5062 inserted at nucleotide position +299 of SCO4284 (transposant cosmid: D95A.2.C09) were introduced into $S$. coelicolor M145 by conjugation from E. coli ET12567/pUZ8002 according to the described protocol [22]. The cosmid used for gene interruption was generated using the in vitro transposition method [22] and obtained from Prof. Paul Dyson (Swansea, UK).

\subsection{Electrophoretic mobility shift assay (EMSA)}

DasR-6His was produced in E. coli BL21(DE3) and purified onto a $\mathrm{Ni}^{2+}$-nitrilotriacetic acid-agarose column as previously described [23]. Pooled fractions with the pure protein were dialyzed overnight at $4{ }^{\circ} \mathrm{C}$ against EMSA buffer (10 mM Tris-Cl, pH 7.5, $1 \mathrm{mM}$ dithiothreitol, $0.25 \mathrm{mM} \mathrm{CaCl}_{2}, 0.5 \mathrm{mM} \mathrm{MgCl}_{2}, 50 \mathrm{mM} \mathrm{KCl}$, and $2 \%$ glycerol). EMSAs were performed using Cy5-labelled dre probes (final concentration, $\sim 0.1 \mu \mathrm{M}$ ) and DasR-6His (final concentration around $1 \mu \mathrm{M}$ ) in a total reaction volume of $50 \mu \mathrm{l}$. Probes were separated by gel electrophoresis in a $1 \%(\mathrm{wt} / \mathrm{vol})$ agarose gel and fluorescent DNA was visualized using a Typhoon Trio + variablemode imager (GE Healthcare). Oligonucleotide used to generate Cy5-fluorescent 40 -bp double-stranded DNA probes $\left(d r e^{\text {nagKA }}\right.$, $d r e^{d a s A}$, and $d r e^{n a g B}$ ) are described in Ref. [23].

\subsection{Western blot}

S. coelicolor ET001 (WT + cosmid D95A.2.C09) and ET002 (QnagA:Tn5062) strains were grown on R2YE and Minimal Media (MM) mannitol plates (with or without GlcNAc 1\%) covered with cellophane discs. S. coelicolor mycelia were scraped with a spatula from the agar plates and frozen at $-70{ }^{\circ} \mathrm{C}$. $50 \mathrm{mg}$ frozen mycelia were suspended into $500 \mu \mathrm{l}$ of extraction buffer (Tris- $\mathrm{HCl} 50 \mathrm{mM}$ $\mathrm{pH} 7.5, \mathrm{MgCl}_{2} 5 \mathrm{mM}, \mathrm{NaCl} 50 \mathrm{mM}, 1 \mathrm{mM}$ DTT, SDS 1\%, and glycerol $2 \%$ ), sonicated for $10 \mathrm{~min}$ with $30 \mathrm{~s}$ pulses (Diagenode, Belgium), and centrifuged at $14,000 \mathrm{rpm}$ for $15 \mathrm{~min}$ (repeated twice). $20 \mu \mathrm{g}$ of proteins were subjected to separation by electrophoresis on a $12 \%$ SDS-PAGE and transferred onto polyvinylidene difluoride membrane (BioRad, Germany) by electroblotting. Membranes were incubated 45 min with primary mouse anti-GFP polyclonal antibodies (Roche) diluted 1:1000 in Tris-buffered saline (TBS) $+0.5 \%$ tween $20+1 \%$ bovine serum albumin. Membranes were washed 3 times 5 min with TBS and eGFP production was visualized using anti-mouse horseradish peroxidase (HRP)-conjugated secondary antibodies and the HRP chemiluminescent detection kit (Bio-Rad).

\section{Results}

\subsection{Multiple effects of phosphorylated (amino)-sugars on the DNA- binding ability of DasR}

Over the years we have performed numerous EMSAs supported by systems wide in vivo analysis of binding sites by ChIP-Chip analysis to identify the new members of the DasR regulon in S. coelicolor. We regularly included a series of assays with GlcN-6P to confirm the previously described inhibitory effect of this phosphorylated aminosugar on the DNA binding of histidine-tagged DasR protein (DasR-6His) [2]. EMSAs made with three different preparations of pure DasR-6His and representative of what we regularly observed, are displayed in Fig. 1 A (see also supplementary Fig. S1). The addition of GlcN-6P to the EMSA reaction mixture simultaneously alters the DasR binding to the fluorescent Cy5labelled DNA probe in two opposite ways i.e., (i) by inhibiting binding to DNA, and (ii) by increasing the retardation of the DasRdre complex such that the complex accumulates into the wells of the agarose gel. The latter is most likely explained by a process called bridging (see below). The inhibition of the DasR DNAbinding activity can also be deduced by the increasing amount of free probe recovered with increasing concentration of GlcN-6P (Fig. 1A). Around 20,000 to 50,000-fold excess of GlcN-6P (compared to the amount of DasR-6His) is required to fully lose the DasR-dre interaction. At these concentrations of GlcN-6P, a fluorescence signal appears in the wells of the agarose gel (Fig. 1A) suggesting that an artefactual high molecular weight DNA-protein complex could be formed (Fig. S1AB).

According to the model deduced from biochemical and crystallographic studies of NagR (the DasR orthologue in B. subtilis), binding of effectors would lead to an extraordinary large conformational change that would lower the DNA-binding ability from a pico-to a nanomolar affinity [19]. This lower affinity would still allow DNA binding by effector-bound dimers where each DasR dimer would contact the DNA via a single DNA-binding domain (Fig. S1B) [19]. This structural rearrangement would lead to the binding of successive probes resulting in a succession of DNA- 

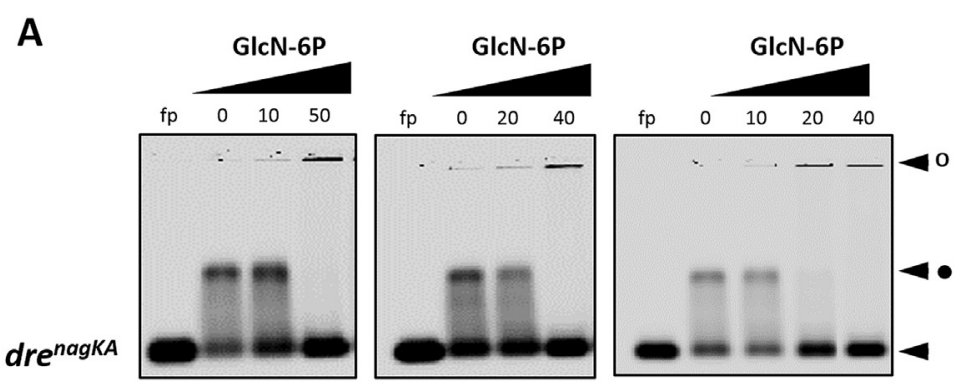

B

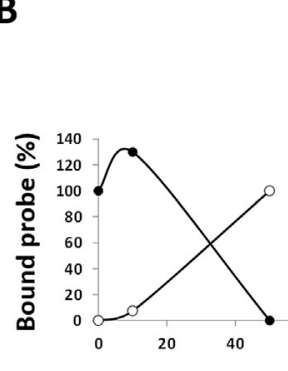

- DasR-dre complex $o$ probe in the well

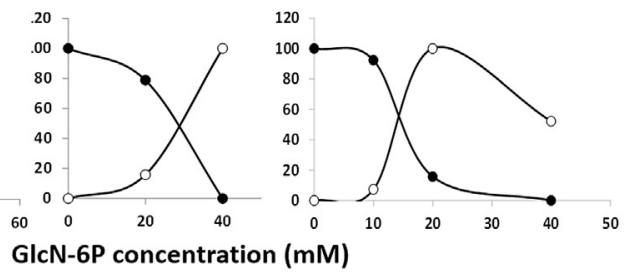

C

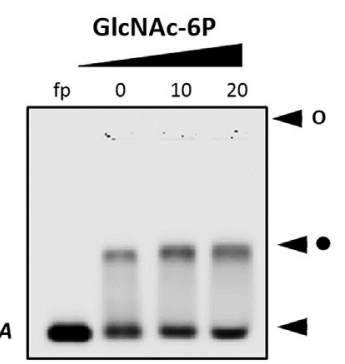

D

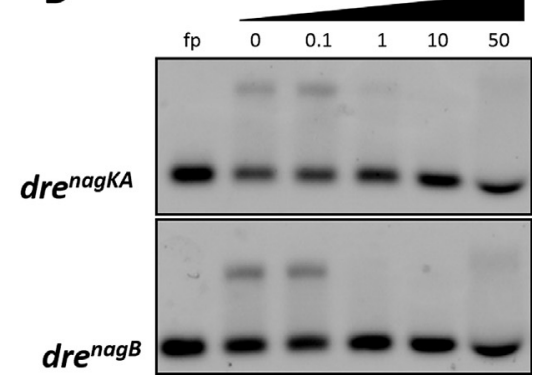

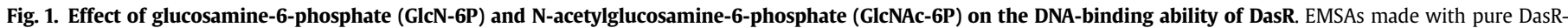

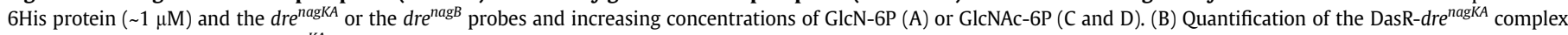

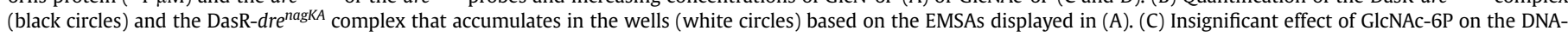

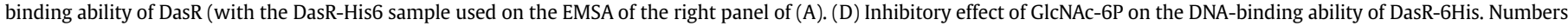
refer to the final concentration of the tested ligand in $\mathrm{mM}$. Abbreviation: fp, free probe.

protein complexes too large to enter the gel and that finally precipitates into the wells (Fig. S1B). The accumulation of DasR-6His into the wells was also observed at high concentrations of the pure protein without any tested ligand added in the EMSA reaction mix (Fig. S1C), a phenomenon that was also previously reported for the NagR protein of $B$. subtilis [18].

In the same manner, we assessed GlcNAc-6P as possible allosteric effector of DasR and in most of our assays we did not observe a significant effect on the retardation of the DasR-dre interacting band (Fig. 1C), exactly as described previously [2]. Surprisingly, with one single preparation of pure DasR-6His, GlcNAc-6P fully prevented DasR binding to dre-containing probes (Fig. 1D). GlcNAc6P inhibited DasR-6His binding to dres at a final concentration of $1 \mathrm{mM}$ in the EMSA buffer which approximately corresponds to 1000 -fold excess of ligand over the pure protein. Using the same sample of pure protein, GlcN-6P prevented the DNA-binding capability of DasR-6His at a final concentration of around $50 \mathrm{mM}$, similar to previous results.

\subsection{Intracellular accumulation of GlcNAc-6P enhances the activity of the nagKA promoter}

The instability of His-tagged DasR preps and the associated problems with reproducibility of in vitro experiments prompted an in vivo assay to investigate the possibility that GlcNAc-6P would also act as an allosteric effector of DasR. The first indirect evidence of a role of GlcNAc-6P in the GlcNAc-mediated response was provided by the examination of the phenotype of the S. coelicolor nagA null mutant $\left(\Delta n a g A^{\mathrm{IFD}}\right.$ (GAM4), $\left.[23,24]\right)$ in which GlcNAc-6P accumulates inside the cytoplasm. As described previously [2], GlcNAc blocks morphological differentiation (sporulation) and antibiotic production of the parental strain S. coelicolor M145 grown on rich R2YE agar plates (see also strains M145 and ET001 in Fig. 2 and Fig. S2). In the current model where the key intracellular signalling molecule is GlcN-6P, one would expect that the nagA mutant would not display the GlcNAc-mediated developmental arrest as GlcNAc$6 \mathrm{P}$ cannot be converted into GlcN-6P in the nagA mutant. However, the $S$. coelicolor nagA mutant does not display a 'neutral' phenotype with regard to the GlcNAc exogenous supply as was observed for the nagE2 mutant (which cannot transport GlcNAc, Fig. 2 [15]). Instead, the nagA mutant shows accelerated timing of aerial hyphae formation and pigmented-antibiotic production (Fig. 2) [24]. Thus, blocking GlcNAc catabolism at the GlcNAc-6P stage (in the nagA mutant) mediates a developmental response which is opposite to when GlcNAc metabolism allows the formation of GlcN-6P.

In order to assess whether the intracellular accumulation of GlcNAc-6P would alter the expression of DasR-controlled genes we used a derivative of knock-out cosmid D95A carrying the Tn5062 transposon) inserted at nucleotide position +299 of nagA (transposant cosmid: D95A.2.C09). In this cosmid, Tn5062 includes the promoterless eGFP reporter gene [22] integrated in the same orientation of the nagA gene (Fig. 2) so that transcription of the gene for eGFP is controlled by the promoter of nagKA (nagKAp), and thus under the control of DasR. Integration of cosmid D95A.2.C09 via a double cross-over will generate apramycin resistant, kanamycin sensitive $\left(\mathrm{Apr}^{\mathrm{R}}\right.$ and $\mathrm{Kan}^{\mathrm{S}}$ ) colonies where the Tn5062 transposon is inserted at nucleotide position +299 of nagA (Fig. 2). Integration of cosmid D95A.2.C09 via a single cross-over will instead lead to apramycin and kanamycin resistant $\left(\operatorname{Apr}^{\mathrm{R}}\right.$ and $\left.\operatorname{Kan}^{\mathrm{R}}\right)$ colonies, with the chromosomal nagA gene intact and an extra-copy of this gene brought by the D95A.2.C09 cosmid and with the eGFP expression under control of the nagKA promoter. In both types of colonies, the accumulation of eGFP can thus serve as a measure of nagKA promoter activity.

The newly obtained nagA mutant (strain ET002, QnagA:Tn5062, abbreviated $\Omega \operatorname{nag} A$ ) also displayed accelerated development upon GlcNAc supply as previously observed with the $S$. coelicolor nagA mutant (GAM4, $\Delta$ nag $A^{\mathrm{IFD}}$ ) generated by in frame deletion (Fig. 2) [23]. A colony resulting from a single cross-over insertion (strain ET001, M145 + D95A.2.C09) was also selected and its phenotype 

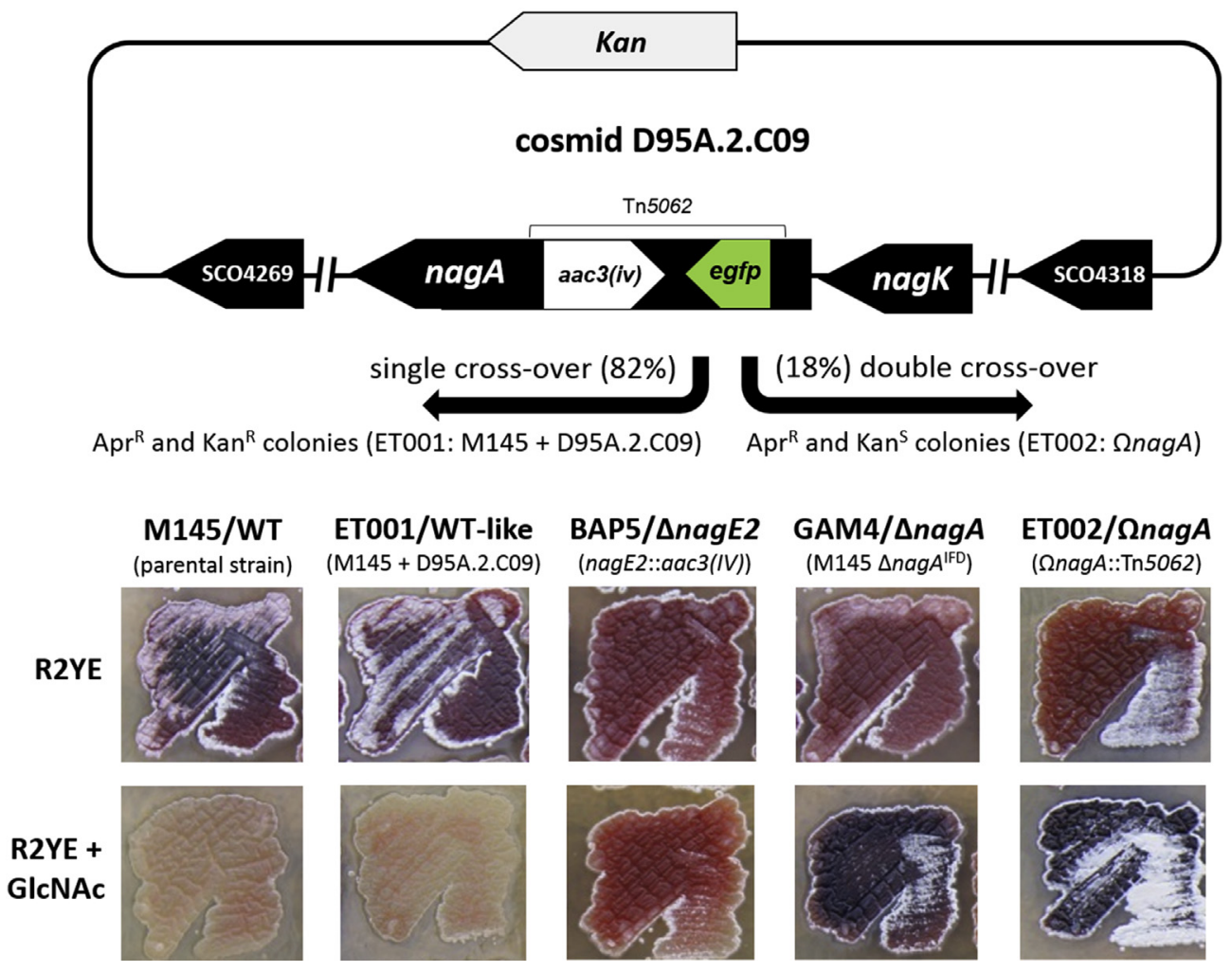

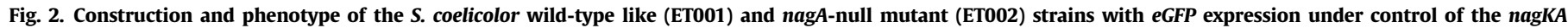

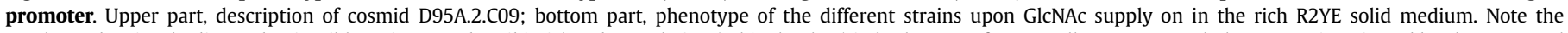

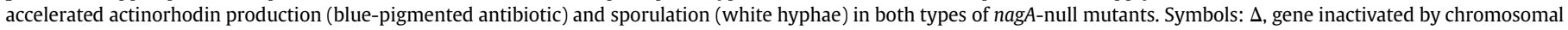
deletion; $\Omega$, gene inactivated by transposon insertion.

was identical to that of the parental strain S. coelicolor M145 (Fig. 2). This demonstrates that the insertion of the entire cosmid has no relevant consequence on the response of $S$. coelicolor to exogenous GlcNAc supply. Quantification of the blue-pigmented antibiotic actinorhodin produced by the strains ET001 (wild-type like S. coelicolor background) and ET002 ( (nnagA) grown on R2YE agar plates with added GlcNAc confirmed that GlcNAc induced the production of actinorhodin (Fig. S2) as a result of the inactivation of $\operatorname{nag} A$.

The transposant @nagA (ET002) and the integrant ET001 (harbouring wt nagA) strains were grown on R2YE or MM mannitol agar plates covered with cellophane discs and mycelia samples were collected after $30,40,48,56,64,72$ and 80 h of growth for total protein extractions. After polyacrylamide gel electrophoresis, the amount of eGFP produced was monitored by Western analysis. This showed that the production of the eGFP was highly increased when strain ET001 was cultivated on R2YE or MM mannitol agar plates supplemented with GlcNAc, confirming the GlcNAc-dependent activity of the nagKA promoter (Fig. 3). A constitutive and high production of eGFP was observed in the nagA mutant ET002, which accumulates GlcNAc-6P intracellularly. The high and constitutive activity of the nagKA promoter in the nagA mutant strongly supports our hypothesis deduced from EMSAs (Fig. 1D) that GlcNAc-6P efficiently relieves the transcriptional repression exerted by DasR. In $E$. coli, the accumulation of GlcNAc-6P as a result of the inactivation of nagA also resulted in the constitutive expression of the GlcNAc utilization regulon controlled by NagC [25].
3.3. The overall phosphate concentration modulates the DasR DNAbinding ability

Triggered by the observation that both GlcN-6P and GlcNAc-6P altered the affinity of DasR for its target sites, we then wondered if other metabolites that are metabolically related could also modulate DasR DNA binding. Fructose-6-phosphate (Fru-6P) was first tested as candidate ligand for DasR as being part of GlcNAc catabolism pathway, namely the product generated by the GlcN-6P deaminase and isomerase NagB. Addition of Fru-6P resulted in higher amounts of the DasR-dre complex whereas the addition of fructose to the EMSA reaction did not affect the ratio of bound/ unbound probe (Fig. 4). Similar enhanced DNA-binding by DasR was also observed with glycerol-3-phosphate (Gly-3P), glucose-1phosphate (Glc-1P) and glucose-6-phosphate (Glc-6P) which are metabolically a few steps away from GlcNAc catabolism (Fig. 4). Importantly, this effect was more drastic with fructose-1,6bisphosphate (Fru-1,6-BP), which suggested that the phosphate content of the ligand tested could be responsible for the improved DNA-binding ability of DasR (Fig. 4). Indeed, the quantification of the bound-probe revealed 2.7 and 1.4-fold more fluorescence signal with $10 \mathrm{mM}$ of Fru-1,6-BP and Fru-6P, respectively, than when the EMSA reaction only contained fructose (Fig. S3). The hypothesis that the phosphate content increases the DNA-binding ability of DasR was confirmed by performing EMSAs with increasing concentrations of sodium phosphate buffer, which resulted in an increase of the bound/unbound-probe ratio. 


\section{ET001 ET002 \\ (WT-like) \\ ( $\Omega$ nagA)}

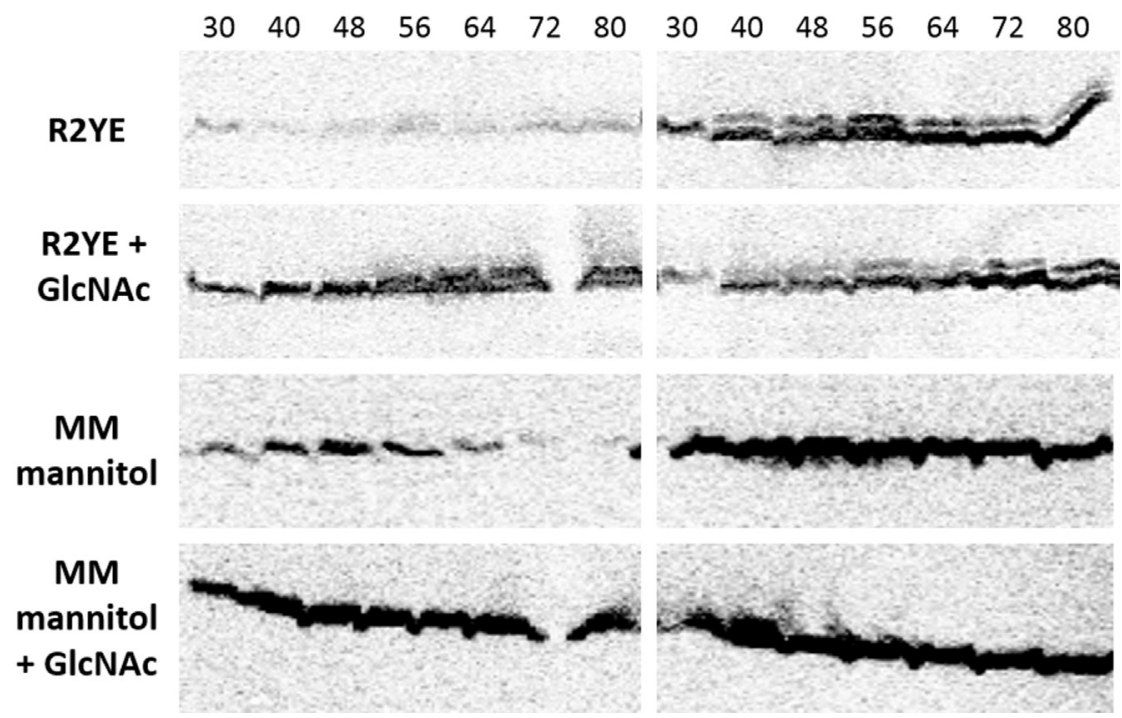

Fig. 3. Effect of the inactivation of nagA on the activity of the nagKA promoter. Visualization of the production of eGFP by immuno-chemiluminescence (see Materials and Methods for details).

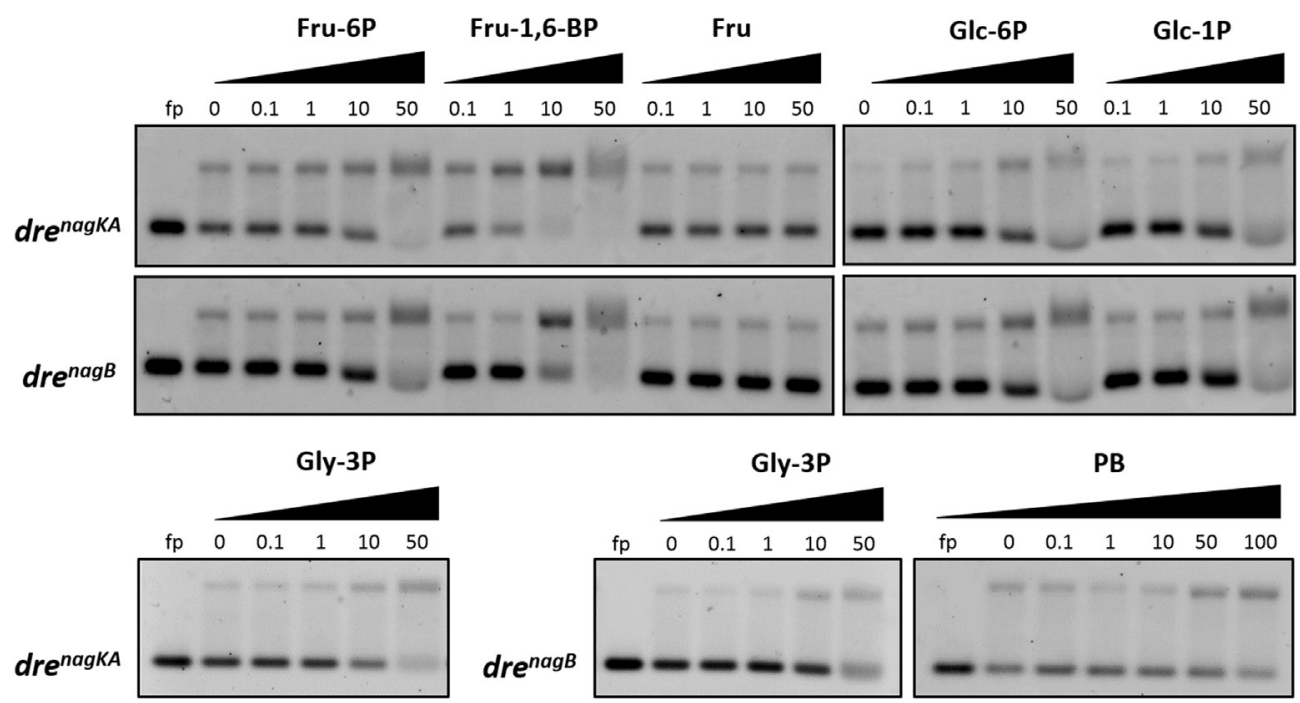

Fig. 4. Effect of phosphorylated sugars and inorganic phosphate on the DNA-binding ability of DasR. For ligand abbreviations please refer to the main text. Numbers refer to the final concentration of the tested ligand in mM. fp, free probe. PB, sodium phosphate buffer pH 7.5.

\section{Discussion}

Our work presents evidence that the mechanism by which externally added GlcNAc induces the expression of DasR-controlled genes is more complex than initially thought. In line with the recent crystallographic and biochemical analyses of the DasR orthologue NagR in B. subtilis [19], GlcNAc-6P (and not only GlcN-6P) could also modulate the DNA-binding ability of DasR. As in S. coelicolor GlcNAc is mainly transported by the PTS, which directly phosphorylates the incoming sugar [14,15], GlcNAc-6P is the first molecule of the GlcNAc catabolism which accumulates intracellularly. It therefore makes sense that DasR unlocks the GlcNAc catabolic machinery in $S$. coelicolor in response to the molecule that first occurs in the cytoplasm, as deduced for GlcNAc utilization control by NagC in
E. coli [25]. GlcNAc catabolism directly connects to glycolysis and is an important source of nitrogen through the activity of NagB, and hence the half-life of GlcN-6P will likely be low. Furthermore, accumulation of GlcN-6P at high concentrations is lethal for S. coelicolor $(26,28)$, which again supports the notion that GlcN-6P is rapidly turned over. Therefore, the very high concentrations of GlcN-6P (20,000-50,000 fold excess relative to the protein) will most likely not be reached in vivo. It is likely that the allosteric effectors have differential effects on DasR in terms of its affinity for the DNA. Indeed, while GlcNAc blocks development of wild-types colonies on R2YE agar plates, it surprisingly led to acceleration of development of nagA null mutants (Fig. 2). The difference between the two strains is that nagA mutants accumulate GlcNAc-6P as shown by preliminary metabolomics analyses (MU, C. Mayer and 
GPvW, unpublished data), while wild-type cells (i.e., with an active GlcNAc deacetylase or NagA) accumulate GlcN-6P. This strongly suggests that DasR-GlcNAc-6P has a different affinity for its in vivo DNA binding sites (dre or otherwise) than DasR-GlcN-6P. This is a very interesting observation that sheds new light on our recent discovery by system-wide in vivo DNA binding studies that DasR does not only bind to dre sites, but also to non-canonical target sites that do not conform to the dre consensus sequence [9]. During normal growth, DasR primarily bound to canonical (Class I) target sites that conformed to the dre consensus sequence, while during development specificity changed, with a strong increase in noncanonical binding events, whereby the latter were not bound in vitro [9]. Unfortunately, our in vitro studies suffered from the fact that not all DasR protein preparations were stable for longer periods of time, which obscured more detailed studies of the affinity of DasR for the different phosphorylated aminosugars (GlcNAc-6P or GlcN-6P). Taken together, we propose that the metabolic status of the cell at a given point in the life cycle will determine the selectivity of DasR in vivo, and hence its effect on the expression of its regulon. The fact that a high concentration of phosphate improves DasR binding to dres also suggests possible cross-talk between phosphate utilization and DasR. As a high intracellular phosphate concentration denotes a high energetic/metabolic state/ activity, the lack of pigmented antibiotic production observed in $S$. coelicolor grown under 'feast' conditions (R2YE + GlcNAc, see Fig. 2 and Fig. S2) could be a response to the increased binding of DasR to the dre associated with actinorhodin and prodiginines biosynthetic clusters. Finally, we postulate that GlcN-6P could play its inhibitory effect on DasR-binding once it arises from Fru-6P in order to make peptidoglycan. GlcN-6P that would come from Fru6P (anabolism) should provide the cell a completely different message than when GlcN-6P would emanate from GlcNAc-6P (catabolism). Further analysis of the effect of the metabolic balance of the cell on the activity and target selectivity of DasR should shed more light on the nutrient- and life cycle-dependent changes of its complex and pleiotropic regulatory network. Such new insights may also facilitate drug-discovery approaches based on the activation of DasR-controlled antibiotic biosynthetic gene clusters.

\section{Acknowledgements}

ET was supported by a FRIA grant and by the Belgian program of Interuniversity Attraction Poles initiated by the Federal Office for Scientific Technical and Cultural Affairs (PAI no. P7/44). MU is supported by a VICI grant (no. 10379) of the Netherlands Technology Foundation STW. SR is an FRS-FNRS research associate.

\section{Appendix A. Supplementary data}

Supplementary data related to this article can be found at http:// dx.doi.org/10.1016/j.bbrc.2015.06.152.

\section{Transparency document}

Transparency document related to this article can be found online at http://dx.doi.org/10.1016/j.bbrc.2015.06.152.

\section{References}

[1] S. Rigali, A. Derouaux, F. Giannotta, J. Dusart, Subdivision of the helix-turnhelix GntR family of bacterial regulators in the FadR, HutC, MocR, and YtrA subfamilies, J. Biol. Chem. 277 (2002) 12507-12515.
[2] S. Rigali, H. Nothaft, E.E. Noens, M. Schlicht, S. Colson, M. Muller, B. Joris, H.K. Koerten, D.A. Hopwood, F. Titgemeyer, G.P. van Wezel, The sugar phosphotransferase system of Streptomyces coelicolor is regulated by the GntRfamily regulator DasR and links $\mathrm{N}$-acetylglucosamine metabolism to the control of development, Mol. Microbiol. 61 (2006) 1237-1251.

[3] S. Colson, J. Stephan, T. Hertrich, A. Saito, G.P. van Wezel, F. Titgemeyer, S. Rigali, Conserved cis-acting elements upstream of genes composing the chitinolytic system of streptomycetes are DasR-responsive elements, J. Mol. Microbiol. Biotechnol. 12 (2007) 60-66.

[4] S. Colson, G.P. van Wezel, M. Craig, E.E. Noens, H. Nothaft, A.M. Mommaas, F. Titgemeyer, B. Joris, S. Rigali, The chitobiose-binding protein, DasA, acts as a link between chitin utilization and morphogenesis in Streptomyces coelicolor, Microbiology 154 (2008) 373-382.

[5] B. Nazari, A. Saito, M. Kobayashi, K. Miyashita, Y. Wang, T. Fujii, High expression levels of chitinase genes in Streptomyces coelicolor A3(2) grown in soil, FEMS Microbiol. Ecol. (3) (2011) 623-635.

[6] C. Liao, S. Rigali, C.L. Cassani, E. Marcellin, L.K. Nielsen, B.C. Ye, Control of chitin and N-acetylglucosamine utilization in Saccharopolyspora erythraea, Microbiology 160 (2014) 1914-1928.

[7] J.W. Seo, Y. Ohnishi, A. Hirata, S. Horinouchi, ATP-binding cassette transport system involved in regulation of morphological differentiation in response to glucose in Streptomyces griseus, J. Bacteriol. 184 (2002) 91-103.

[8] B. Nazari, M. Kobayashi, A. Saito, A. Hassaninasab, K. Miyashita, T. Fujii, Chitininduced gene expression in secondary metabolic pathways of Streptomyces coelicolor A3(2) grown in soil, Appl. Environ. Microbiol. 79 (2013) 707-713.

[9] M.A. Swiatek-Polatynska, G. Bucca, E. Laing, J. Gubbens, F. Titgemeyer, C.P. Smith, S. Rigali, G.P. van Wezel, Genome-Wide Analysis of In Vivo Binding of the Master Regulator DasR in Streptomyces coelicolor Identifies Novel NonCanonical Targets, PLoS One 10 (2015) e0122479.

[10] S. Rigali, F. Titgemeyer, S. Barends, S. Mulder, A.W. Thomae, D.A. Hopwood, G.P. van Wezel, Feast or famine: the global regulator DasR links nutrient stress to antibiotic production by Streptomyces, EMBO Rep. 9 (2008) 670-675.

[11] M. Craig, S. Lambert, S. Jourdan, E. Tenconi, S. Colson, M. Maciejewska, M. Ongena, J.F. Martin, G. van Wezel, S. Rigali, Unsuspected control of siderophore production by $\mathrm{N}$-acetylglucosamine in streptomycetes, Environ. Microbiol. Rep. 4 (2012) 512-521.

[12] S. Lambert, M.F. Traxler, M. Craig, M. Maciejewska, M. Ongena, G.P. van Wezel, R. Kolter, S. Rigali, Altered desferrioxamine-mediated iron utilization is a common trait of bald mutants of Streptomyces coelicolor, Metallomics 6 (2014) 1390-1399.

[13] M.F. Traxler, M.R. Seyedsayamdost, J. Clardy, R. Kolter, Interspecies modulation of bacterial development through iron competition and siderophore piracy, Mol. Microbiol. 86 (2012) 628-644.

[14] H. Nothaft, D. Dresel, A. Willimek, K. Mahr, M. Niederweis, F. Titgemeyer, The phosphotransferase system of Streptomyces coelicolor is biased for N-acetylglucosamine metabolism, J. Bacteriol. 185 (2003) 7019-7023.

[15] H. Nothaft, S. Rigali, B. Boomsma, M. Swiatek, K.J. McDowall, G.P. van Wezel, F. Titgemeyer, The permease gene nagE2 is the key to N-acetylglucosamine sensing and utilization in Streptomyces coelicolor and is subject to multi-level control, Mol. Microbiol. (5) (2010) 1133-1144.

[16] M. Resch, E. Schiltz, F. Titgemeyer, Y.A. Muller, Insight into the induction mechanism of the GntR/HutC bacterial transcription regulator YvoA, Nucleic Acids Res. 38 (2010) 2485-2497.

[17] R. Bertram, S. Rigali, N. Wood, A.T. Lulko, O.P. Kuipers, F. Titgemeyer, Regulon of the N-Acetylglucosamine Utilization Regulator NagR in Bacillus subtilis, J. Bacteriol. 193 (2011) 3525-3536.

[18] I. Gaugue, J. Oberto, J. Plumbridge, Regulation of amino sugar utilization in Bacillus subtilis by the GntR family regulators, NagR and GamR, Mol. Microbiol. 92 (2014) 100-115.

[19] S.B. Fillenberg, F.C. Grau, G. Seidel, Y.A. Muller, Structural insight into operator dre-sites recognition and effector binding in the GntR/HutC transcription regulator NagR, Nucleic Acids Res. 43 (2015) 1283-1296.

[20] T. Kieser, M. Bibb, M.J. Butner, K.F. Chater, Practical Streptomyces Genetics, John Innes Foundation, Nowich, UK, 2000.

[21] R.D.W, J. Sambrook, , Molecular Cloning: a Laboratory Manual, third ed., Cold Spring Harbor Laboratory Press, New York (, 2001.

[22] A. Bishop, S. Fielding, P. Dyson, P. Herron, Systematic insertional mutagenesis of a streptomycete genome: a link between osmoadaptation and antibiotic production, Genome Res. 14 (2004) 893-900.

[23] M.A. Swiatek, E. Tenconi, S. Rigali, G.P. van Wezel, Functional analysis of the $\mathrm{N}$-acetylglucosamine metabolic genes of Streptomyces coelicolor and role in control of development and antibiotic production, J. Bacteriol. 194 (2012) 1136-1144.

[24] M.A. Swiatek, M. Urem, E. Tenconi, S. Rigali, G.P. van Wezel, Engineering of Nacetylglucosamine metabolism for improved antibiotic production in Streptomyces coelicolor $\mathrm{A} 3(2)$ and an unsuspected role of NagA in glucosamine metabolism, Bioengineered 3 (2012) 280-285.

[25] J.A. Plumbridge, Repression and induction of the nag regulon of Escherichia coli $\mathrm{K}-12$ : the roles of $\operatorname{nagC}$ and nagA in maintenance of the uninduced state, Mol. Microbiol. 5 (1991) 2053-2062. 